\title{
Appropriate Architecture and Urban Design Research Centre for Creative Thinking and Communication
}

\author{
Mona Bisadi, Farhang Mozaffar, Seyed Bagher Hosseini \\ School of Architecture and Urban Studies, \\ Iran University of Science and Technology, 16846-13114, 7ehran, Iran \\ bisadi.mona@gmail.com
}

\begin{abstract}
Progressive research activities, assess entail factors in future societies, are strongly dependent on the creativity and innovation. This paper considers the built environment as one of the influential aspect in creativity and innovation and seeks its appropriate features. Among various choices, we adopt academic research centers of architecture and urban design for the sake of this study. At first, we identify four effective spatial characteristics, including privacy, beauty, spatial diversity, and proximity. Then, we conduct the analysis based on the survey method and, ultimately, we propose some design patterns in order to enhance the researchers' creativity and innovation.
\end{abstract}

Keywords: Architecture and urban design research center; creativity; innovation; spatial characteristics.

eISSN 2514-751X @ 2018. The Authors. Published for AMER ABRA cE-Bs by e-International Publishing House, Ltd., UK. This is an open access article under the CC BY-NC-ND license (http://creativecommons.org/licenses/bync-nd/4.0/). Peer-review under responsibility of AMER (Association of Malaysian Environment-Behaviour Researchers), ABRA (Association of Behavioural Researchers on Asians) and cE-Bs (Centre for EnvironmentBehaviour Studies), Faculty of Architecture, Planning \& Surveying, Universiti Teknologi MARA, Malaysia.

DOI: https://doi.org/10.21834/aje-bs.v3i7.253 


\subsection{Introduction}

Evidently, research is an extremely vital elementin the development path of asociety. Research is the process of solving current problems and answering to the novel questions through viable and reliable methods, and creativity is a key factor in this process (Gobo et al., 2004). Universities, research and development departments (R\&Ds), and research centers are main contexts for research activities. The progression of each organization requires transition from a present state toward a desired future state. Creativity and innovation are psychological processes facilitating such transformations (Rank et al., 2004).

Within the creativity research literature, Rhodes (1961) categorized creativity into influential factors of persons, processes, products, and places. In the current paper, the focus is on the place (environment). Jon Lang (1987) stated that architects create potential environment and the way that individuals use the place is the effect of environment on them. The specific behaviors would not necessarily happen if the environment can afford them. However, if the place does not have the capacity of doing specific behaviors, these behaviors would never happen. Thus, the environment should be programmed to encourage desirable behaviors (Lang, 1987). As the built environment affects the human behaviors, physical setting could impact on individuals' creativity and innovation (Amabile, 1996; Martens, 2011; Woodman et al., 1993). Hence, to increase creativity and innovation in the field of research, it is necessary to design an appropriate research place where facilitates researchers' needs and encourages them to be more creative and innovative.

So far, a lot of researchers have studied the effect of built environment on creativity and innovation in contexts of educational places such as kindergartens and schools (e.g. Mozaffar, 1997; Niu, 2007; Shafaie \& Madani, 2010), urban and residential areas (e.g. Brodersen, 2005; Faizia et al., 2012), and workplaces (e.g. Amabile,1999; Dul \& Ceylan, 2011; Martens, 2011; McCoy \& Evans, 2002). Although the role of creativity and innovation in research centers is of vital importance, the literatures in this area are just limited to the studies of Toker (2003) and Toker and Gray (2008). These studies focused on innovation in university research centers and supposed that communication and face-to-face consultation are the main sources of innovation. They analyzed the layout of research offices and labs associated with six university research centers, categorized them into three arrangements, and introduced one of them as the best arrangement in increasing innovative outcomes. In addition to the configuration of spaces of a plan, the spatial characteristics of built environment have an impact on researchers' behaviors.

The goal of the current research is to identify the effective spatial characteristics of a research center that cause the growth of researchers' creative and innovative outcomes. Additionally, we investigate that whether there is any difference between the impact of those spatial characteristics on the creativity and innovation. As well as, we devise architectural approaches to realize the featured spatial aspects.

\subsection{Methodology}

\section{Setting and Participants}


As a specific setting, we chose "university architecture and urban design (UAUD) research center" because architects and urban designers are highly sensitive to the built environment and they are more familiar with spatial phrases and concepts. Therefore, we selected the sample group among faculties and $\mathrm{PhD}$ students of architecture and urban design school of the universities in Tehran, Iran.

\section{Procedures}

In order to achieve the research objectives, we use the survey method for data collection. The present research has two steps. First, finding the appropriate spatial characteristics of research centers in increasing researchers' creative and innovative outcomes. Second, evaluating the impact of each spatial characteristics achieved from the previous step on researchers' creative thinking and communication since creative thinking is the most influential factor in creativity and communication is the most effective factor in innovation. In the first step, the Delphi method applied. We collected the data by the help of an open-ended questionnaire filled out by 12 expert architectures in the field of creativity and innovation (6 academicians and 6 practitioners). In the questionnaire, we asked the experts about the appropriate research center that could increase the researchers' creativity and innovation. In the second step, we evaluated the effects of derived spatial characteristics on researchers' creative thinking and communication. To do this, we classified the spaces of research center into private offices and common areas which we studied them separately. We collected the required data of this stage by a closed-ended questionnaire in four-point Likert scale ${ }^{*} .90$ faculties and PhD students of architecture and urban design filled out the questionnaire and we analyzed the collected data by the method of $t$-test.

\subsection{Results and Discussions}

We analyzed the answers of the open-ended questionnaire, from the first step of the present research, with the objective of reaching the spatial characteristics of aresearchcenterinorder to increase researchers' creativity and innovation. The obtained spatial characteristics include four items: privacy, beauty, spatial diversity, and proximity. Note that the latter item refers to the researchers' proximity.

In the second step, we applied the statistics t-test on the collected questionnaire-based data in offices and common areas. Then, we initially discussed the obtained results mostly from the mathematical point of view. Thereafter, we presented the architectural inferences drawn from the numerical analysis.

\section{Offices}

Table 1 shows the mean, standard deviation, and standard error of mean of the effect of each spatial characteristic on creative thinking and communication in offices. Table 2 reports the degree of freedom, two-tailed probability (The probability value $(p)$, which is in the column labeled Sig., corresponds to a value of $t$ as big as obtained that could occur if the null hypothesis were true (i.e. there is no difference between these means), and test statistic, $t$. 
Referring to Table 2, there is no significant difference between the effect of privacy of offices on creative thinking and communication as $p>.05$. On the other hand, Table 1 shows the means of creative thinking $(M=3.46, S E=.057)$ and communication $(M=3.29, \mathrm{SE}=.069)$ that are both greater than 3 . Therefore, it can deduce that architecture and urban design researchers agree with the effect of office privacy on increasing their creative thinking and communication. Therefore, this spatial aspect deserves an extremely high priority in the design process of researchers' offices.

On average, the beauty of offices impacts creative thinking $(M=3.39, \mathrm{SE}=.066)$ significantly higher than communication $(M=2.66, S E=.073), \mathrm{t}(89)=7.87, p<.001$ and $r$ $=.64$ that shows a large effect. Moreover, based on $t(89)=8.57, p<.001$, the spatial diversity in offices affects creative thinking $(M=2.98, S E=.045)$ significantly higher than communication $(M=2.24, S E=0.83)$ with a large effect $(r=.67)$. The mean of communication under the influence of spatial diversity shows that architecture and urban design researchers do not agree with the positive effect of this spatial characteristic on communication. It is same to the effect of researchers' proximity in their offices on creative thinking $(M=2.12, S E=.067)$. Besides, researchers' proximity affects communication ( $M=$ $3.10, S E=.061$ ) significantly higher than creative thinking, $\mathrm{t}(89)=-13.20, p<.001$. In this case, $r=.81$ represents a large effect.

Table 1: The Influence of Spatial Characteristics of Offices on Researchers'

Creative Thinking and Communication

\begin{tabular}{llllll}
\hline Spatial characteristics & Monitored factor & Mean & $\mathrm{N}$ & Std. Deviation & Std. Error Mean \\
\hline Privacy & Creative thinking & 3.46 & 90 & .54 & .057 \\
\cline { 2 - 6 } Beauty & Communicati on & 3.29 & 90 & .66 & .069 \\
\multirow{3}{*}{ Spatial diversity } & Creative thinking & 3.39 & 90 & .63 & .066 \\
\cline { 2 - 6 } Proximity & Communication & 2.66 & 90 & .69 & .073 \\
& Creative thinking & 2.98 & 90 & .42 & .045 \\
\cline { 2 - 6 } & Communication & 2.24 & 90 & .78 & .083 \\
& Creative thinking & 2.12 & 90 & .63 & .067 \\
\cline { 2 - 5 } & Communication & 3.10 & 90 & .58 & .061 \\
\hline
\end{tabular}

Table 2: Comparing the Means of Creative Thinking and Communication on the Influence of Spatial Characteristics of Offices

\begin{tabular}{llll}
\hline $\begin{array}{l}\text { Spatial } \\
\text { characteristics }\end{array}$ & $\mathrm{t}$ & df & Sig (2-tailed) \\
\hline Privacy & 1.89 & 89 & .062 \\
Beauty & 7.87 & 89 & .000 \\
Spatial diversity & 8.57 & 89 & .000 \\
Proximity & -13.20 & 89 & .000 \\
\hline
\end{tabular}




\section{Common Areas}

Table 3 presents the means, standard deviations, and standard errors of mean values associated with the influence of spatial characteristics of common areas on researchers' creative thinking and communication. Table 4 shows the results of t-tests.

According to Tables 3 and 4 , having privacy in common areas has influence oncreative thinking $(M=3.37, S E=.058)$ significantlyhigherthan communication $(M=3.17, S E=.056), t(89)$ $=2.52, p<.05$. The associated $r=.26$ demonstrates a medium effect. Beauty of common areas impacts researchers' creative thinking $(M=3.37, \mathrm{SE}=.058)$ significantly higher than communication $(M=3.12, S E=.047), \mathrm{t}(89)=3.61, p<.001$ with a medium effect $(r=.36)$.

Table 3: The Influence of Spatial Characteristics of Common Areas on Researchers' Creative Thinking and Communication

\begin{tabular}{cccccc}
\hline \multirow{2}{*}{$\begin{array}{c}\text { Spatial } \\
\text { characteristics }\end{array}$} & \multicolumn{1}{c}{ Mean } & N & Std. Deviation & Std. Error Mean \\
\hline \multirow{2}{*}{ Privacy } & \multicolumn{2}{c}{ Creative thinking 3.37 } & 90 & .55 & .058 \\
\cline { 2 - 5 } Beauty & Communication & 3.17 & 90 & .53 & .056 \\
& \multicolumn{2}{c}{ Creative thinking 3.37 } & 90 & .55 & .058 \\
\cline { 2 - 5 } Spatial diversity & Communication & 3.12 & 90 & .44 & .047 \\
& $\quad$ Creative thinking 2.93 & 90 & .51 & .054 \\
\cline { 2 - 5 } Proximity & Communication & 3.19 & 90 & .56 & .059 \\
& \multicolumn{2}{c}{ Creative thinking 2.68 } & 90 & .72 & .075 \\
\cline { 2 - 5 } & Communication & 3.12 & 90 & .73 & .077 \\
\hline
\end{tabular}

Table 4: Comparing the Means of Creative Thinking and Communication on the Influence of Spatial Characteristics of Common Areas

\begin{tabular}{llll}
\hline $\begin{array}{l}\text { Spatial } \\
\text { characteristics }\end{array}$ & $T$ & Df & Sig (2-tailed) \\
\hline Privacy & 2.52 & 89 & .013 \\
Beauty & 3.61 & 89 & .000 \\
Spatial diversity & -4.61 & 89 & .000 \\
Proximity & -5.13 & 89 & .000 \\
\hline
\end{tabular}

Spatial diversity in common areas affects communication $(M=3.19, S E=.059)$ significantly greater than creative thinking $(M=2.93, S E=.054), t(89)=-4.61, p<.001$, and $r$ $=.44$ shows a medium effect. Researchers' proximity affects communication $(M=3.12, S E$ $=.077)$ in common areas significantly higher than creative thinking $(M=2.68, S E=.075)$, $t(89)=-5.13, p<.001$, and $r=.47$, which is almost a large effect.

\section{Discussions}

The limitation acknowledged by the authors is that the number of Architecture and Urban Design Research Centers is extremely few. Therefore, the similarities between this type of 
place and educational contexts leads us to focus on universities where the place of research and education are. In this case, we chose faculties and PhD students of architecture and urban design as a sample group.

As a matter of fact, creative thinking needs solitude while innovation is based on communication. Thus, we supposed that offices should support creative thinking more than communication; and common areas should facilitate communication more than creative thinking. Influential spatial characteristics in increasing creative thinking in offices are more serious than features encouraging communication, and in common areas this relation is vice versa. Consequently, offices should be beautiful and diverse as well as be private. Fig. 1 shows a sample of the office with theses spatial characteristics. Besides, the commonareas such as a lobby, lounge, corridor, coffee shop/restaurant, gathering room, and team work room should be designed diversely and also in a way that increases researchers' proximity and visibility to each other. Figs. 2, 3, and 4 show a number of sketches of common areas with the mentioned aspects.

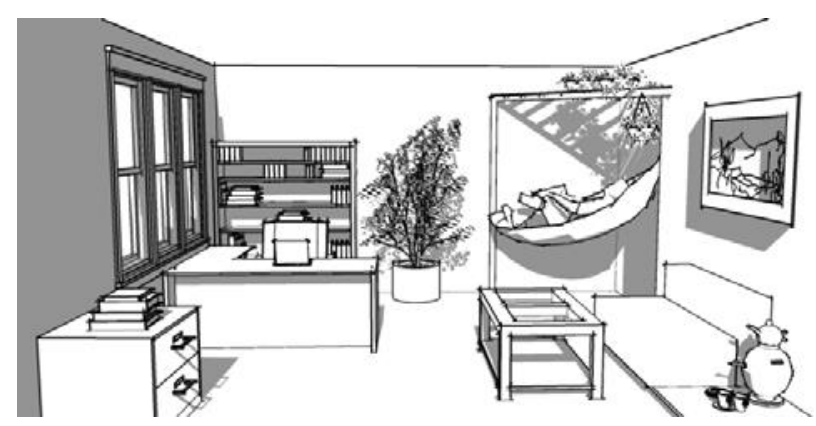

Figure 1: The Sketch of a Diverse Office in UAUD Research Center

In Fig.1, researchers could cover wider domain of their need, including study and work behind the desk, having coffee on the sofa, and short resting on the hammock.

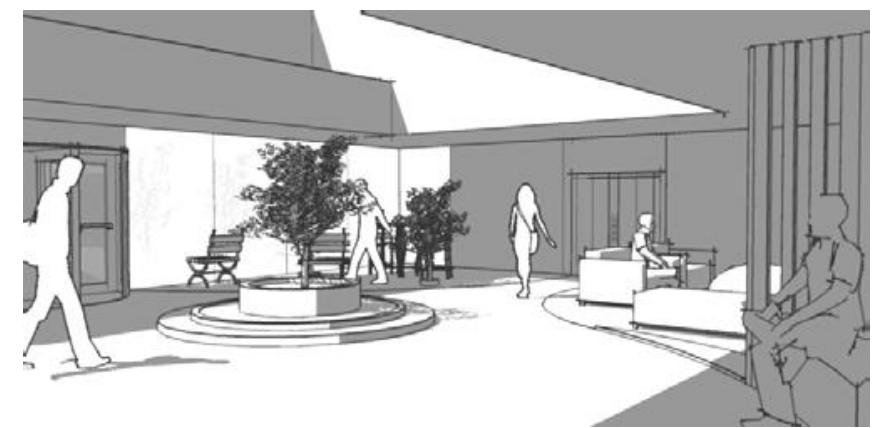

Figure 2: The Sketch of Diverse CommonArea(lobby) Where Provides Various Types of Sitting Zones 
In Fig. 2, the lobby is an integrated space where increases the sense of visibility and proximity. In this sample, connection of outside landscape to the indoor green space is effective in growth of variety and visibility of the place.

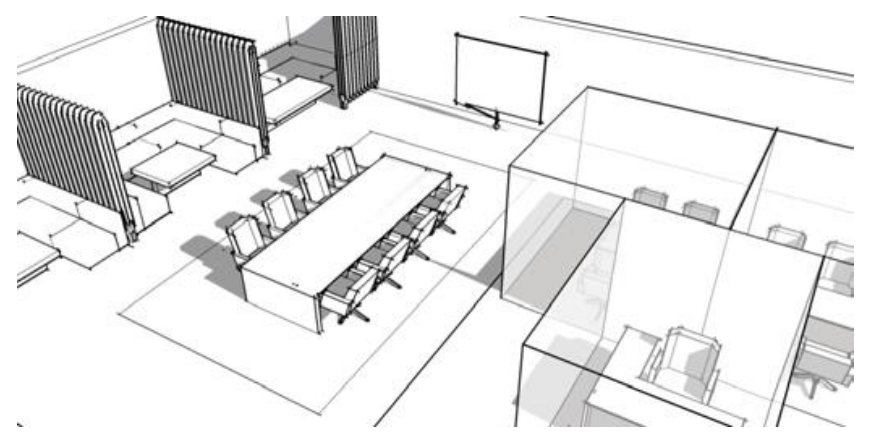

Figure 3: The Sketch of Team Work Room Designed Variously

In the mode in Fig. 3l, researchers have the opportunity to work in acoustic glass cubes where they could cover the walls by blinds to have no visibility to outside. In these cubes, small groups could individually work. In the left hand side of the room, a large group that is the combination of some subgroups could work. In this model, each subgroup can have own place for inter-subgroup interaction along with a place for intra-subgroup consultation.

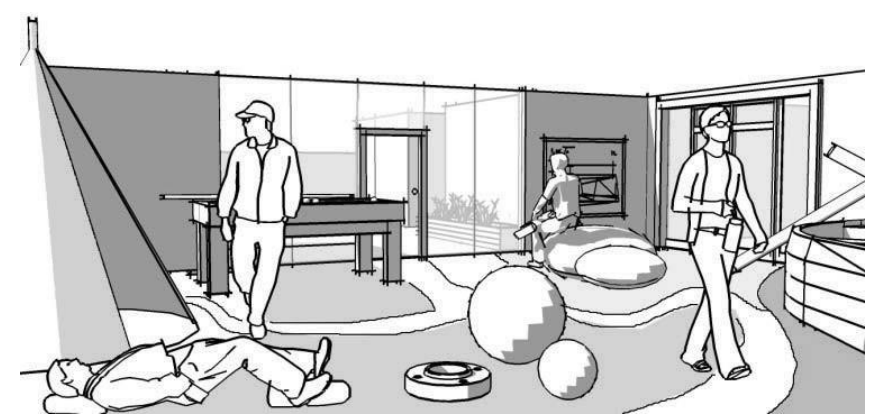

Figure 4: The Sketch of a Probable Lounge in UAUD Research Center

The furniture in Fig. 4 could be playing tools such as a pool table, balls, a hand-football and other features such as a tent for resting in and having a bit more solitude, a micro kitchen for having a snack and coffee during a day, and a whiteboard for spur-of-the-moment brainstorming. This space could restore researchers' energy, motivation, and attention since it is a various attracting room for rest, think, and communicate.

Based on the obtained results, beautiful environment could increase creative thinking in both private and common areas. Also, researchers' proximity in both areas could encourage innovation since seeing other colleagues encourages them to interact and communicate. 
On the other hand, researchers do not agree with sharing an office with other colleagues as it may decline their creative thinking; while, they agree that researcher's proximity in offices could increase their communication. As a solution of this contradictory matter, it is possible to settle researchers in independent offices that are proximate to each other. Therefore along with preserving their privacy, whenever they leave their office, they could see other researchers. Based on the obtained results, beautiful environment could increase creative thinking in both private and common areas. Also, researchers' proximity in both areas could encourage innovation since seeing other colleagues encourages them to interact and communicate. On the other hand, researchers do not agree with sharing an office with other colleagues as it may decline their creative thinking; while, they agree that researcher's proximity in offices could increase their communication. As a solution of this contradictory matter, it is possible to settle researchers in independent offices that are proximate to each other. Therefore along with preserving their privacy, whenever they leave their office, they could see other researchers.

\subsection{Conclusion}

The results showed that the influential spatial characteristics for increasing researchers' creative and innovative outcomes which should be considered in UAUD research centers were privacy, beauty, spatial diversity, and proximity. In order to improve architecture and urban design researchers' creativity and innovation, offices should be beautiful and diverse and also have privacy. Additionally, common areas should be designed diversely and provide researchers' proximity. We draw more detailed architectural remarks in the following separately for private offices and common areas.

\section{In Offices}

According to the analysis, we derive the design patterns of offices in UAUD research center that can increase creative and innovative outcomes, as below:-

1) Locating individual offices close to each other as a colony: it could increase the chance of interaction among researchers while respecting their privacy, simultaneously. The reason is that the common path to the offices increases the rate of unprogrammed conversation and interaction. These interactions might frequently occur in front of elevator, stairs, and corridors. Moreover, individual offices support researchers' privacy by supplying their own territories that is essential in researchers' creative thinking and also their communication.

2) Decorating offices by natural elements such as plants and natural material such as wood or stone: beauty of natural elements in interior design of offices leads to the pretty workplaces that facilitate researchers' creative thinking.

3) Having window in offices: the window in the office, in addition to supplying natural light, could increase the beauty as well as diversity of places - if the view of the window is appropriate.

4) Creating diverse spaces in offices by the help of natural elements, colors, and furniture: diversity in offices could answer the wider domain of researchers' needs and tastes. This aspect is helpful in thinking creatively due to the heightening researchers' tranquility and 
physical comfort. Fig. 1 shows a sample of this pattern.

\section{In Common Areas}

The design patterns of common areas in UAUD research center, based on the analysis, could be outlined as follows:-

1) Connectedness and continuity of open and closed spaces to enhance visibility and the sense of proximity: it could help researchers to meet each other more often in common areas and increase their unprogrammed communication. This aspect leads to a dynamic place where could increase innovative outcomes by facilitating researchers' interaction and communication. Fig. 2 shows a model of this pattern.

2) Usage of indoor plants in common areas: usage of plants and green spaces are one of the best ways for making pleasured spaces. Generally speaking, nature increases humans' tranquility and helps them to think more creatively as well as improves their attention.

3) Designing specific places for gathering, chatting, playing, and exercising: Fig. 4 shows a sample of this place. This pattern could increase researchers' liveliness and could restore their attention. In this type of an unofficial place, researchers can have fun along with interaction with each other. This kind of diversity in types of spaces is accompanied by attractiveness that would increase researchers' creativity and innovation.

4) Creating diverse spaces in common areas- such as coffee shop/ restaurant, lobby, corridors, and gathering area- by the help of natural elements, colors, light, and furniture: it could satisfy wider group of people with various tastes. For example, as shown in Fig. 2, if someone prefers to sit alone, she/he could choose single seats designed individually. Also, if someone wants to chat and communicate with other ones, she/he could choose seats laid out in the colony.

5) Designing some subspaces in group work rooms- to let a group simultaneously work in some subgroups: it could be possible by arrangement of furniture and also using partitions (it should be considered that visibility of spaces preserved such as Fig. 3). This aspect increases researchers' proximity during team work and lets the group divide into subgroups -if necessary- while each subgroup has its own separate place. It would increase group outcomes by facilitating communication inter-subgroups as well as intra-ones.

In order to achieve more practical details, in future study, one can synthesize virtual spaces based on the above guidelines and can examine the researchers' creativity, by specific test, with respect to the alteration of design aspects. Furthermore, a similar analysis could be conducted on researches of other majorities such as engineering, medicine, humanities, art, etc to investigate how their environmental perceptions influence the architectural design outcomes and to see if there is any difference between the creative and innovative workplace for architecture/urban design researchers and other majors' researchers.

\section{References}

Amabile, T. M. (1996). Creativity in context: Update to the social psychology of creativity. Boulder, CO:Westview. 
Amabile T. M., Conti, R., Coon, H., Lazenby J., \& Herron M. (1996). Assessing the workenvironment for creativity. Academy ofManagement Journal, 39, 1154 - 1184.

Brodersen, R. M. ( 2005). Environmental effects on creative thinking and the role of affect, arousal, and personenvironment ßt. Ph.D. Dissertation, Colorado State University.

Dul, J. \& Ceylan, C. (2011). Work environments for employee creativity. Ergonomics, 54(1), 12 - 20

Faizi, M., Karimi Azaria, A., \& Norouzian Maleki, S. (2012). Design Principles of Residential Spaces to Promote Children's Creativity, Procedia - Social and Behavioral Sciences, 35, 468 - 474

Gobo, G., Seale, C., Silverman, D., \& Gubrium, J. F. (2004). Qualitative Research Practice. London: SAGE publications.

Lang, J. T. (1987). Creating Architectural Theory: The Role of the Behavioral Sciences in Environmental Design. New York: John Wiley \& Sons Inc.

MartensY.(2011). Creativeworkplace: instrumental and symbolicsupport for creativity. Facilities, 29 (1/2), 63 - 79.

Mozaffar, F. (1997). A suggested approach for school design based on psychological and communication theories, for Iran. Doctoral dissertation, Sheffield University.

Niu, Weihua. (2007). Individual and Environmental Influences on Chinese

Student Creativity. Journal of Creative Behavior, 41 (3), 151 - 175.

Rhodes, M. (1961). An analysis of creativity. Phi Delta Kappan, 42(7), 305 - 310.

Rank J., Pace V.L., \& Frese M. (2004). Three Avenues for Future Research on Creativity, Innovation, and Initiative. APPLIED PSYCHOLOGY: AN INTERNATIONAL REVIEW, 53(4), $518-528$.

Toker U (2003). Space for Innovation: Effects of Space on Innovation Processes in Basic Science and Research Settings. A dissertation submitted to the Graduate Faculty of North Carolina State University in partial fulfillment of the requirements for the Degree of Doctor of Philosophy, North Carolina.

Toker, U. \& Gray, D.O. (2008). Innovation spaces: workspace planning and innovation in US university research centers, Research Policy, 37, 309 - 329.

Woodman R.W., Sawyer J.E., \& Griffin R.W. (1993). Toward a theory of organisational creativity, Academy of Management Review, 18(2), 293 - 321. 